\title{
TRENISSIMO: IMPROVING THE MICROSCOPIC SIMULATION OF RAILWAY NETWORKS
}

\author{
STEFANO DE FABRIS, GIORGIO MEDEOSSI \& GIULIANO MONTANARO \\ TRENOlab, Italy
}

\begin{abstract}
Railway is a complex system. Infrastructure, rolling stock and timetable interact together with human factors and daily disturbances. Microscopic simulation is the most accurate technique to model and quantitatively represent these complex interactions and support railway undertakings at all stages of planning. The most widely-used simulation tools were developed several years ago and have since then maintained their original principles. But, in the era of Big Data the availability of large amounts of extremely accurate train tracking data and the increased computational power allows modelling reality at a higher level of detail without exceeding the reasonable computation times. The new trenissimo tool was developed with the specific aim of obtaining a more realistic simulation of railway operations, combining new characteristics and functionalities not yet or only in part implemented in the existing tools. One of the most important innovations is the representation of both drivers and dispatchers as agents, who respectively drive the trains following the timetable and signalling/ATP system and control them based on given criteria or algorithms. A second characteristic is the control of signals: as in the reality, the block signals are automatically switched to green, while the others are operated by the dispatchers. A third is the possibility to consider in real time the impact of the number of passengers waiting at the platform on the dwell time of trains, and thus correctly represent their effect, especially on high-frequency services. The paper describes in detail these innovative functions and shows the results of the first tests performed on two lines.
\end{abstract}

Keywords: railway simulation, railway planning, railway operations modelling, calibration.

\section{INTRODUCTION}

Rail operations are the result of a complex interaction of infrastructure and signaling, rolling stock and timetable. This interaction is further complicated by a number of human factors and other unpredictable phenomena and stochastic disturbances. This complexity, combined with the high costs of infrastructure and its low flexibility, forces planners to design the system carefully.

The first step in this planning process is to understand real operations through the analysis of railway traffic to identify disturbances and critical points, which often lead to simple improvements that may increase the performances of the system.

After this initial step, simulation is used for estimating ex-ante the capacity and the corresponding reliability. The same tool used for analyzing real traffic might be used also to compare reality and simulation of multiple scenarios.

Consequently, rail planning can be viewed as a loop (Fig. 1), which starts from the problem definition and whose elements are the analysis of real data, the design of scenarios (which might include infrastructure, rolling stock and timetable alternatives), the simulation and the analysis of simulated results compared to the aim of the project. Smart interfaces are required to avoid most manual steps and therefore allow a seamless integration among the tools: when all datasets are integrated, not only the time required to perform a study shrinks remarkably, but also the level of detail improves.

The loop is the ideal support to timetable planning, which is becoming more demanding since punctuality has become fundamental since delays lead to higher costs and lower customer satisfaction. Additionally, in particular on complex or heavily used networks, it is 


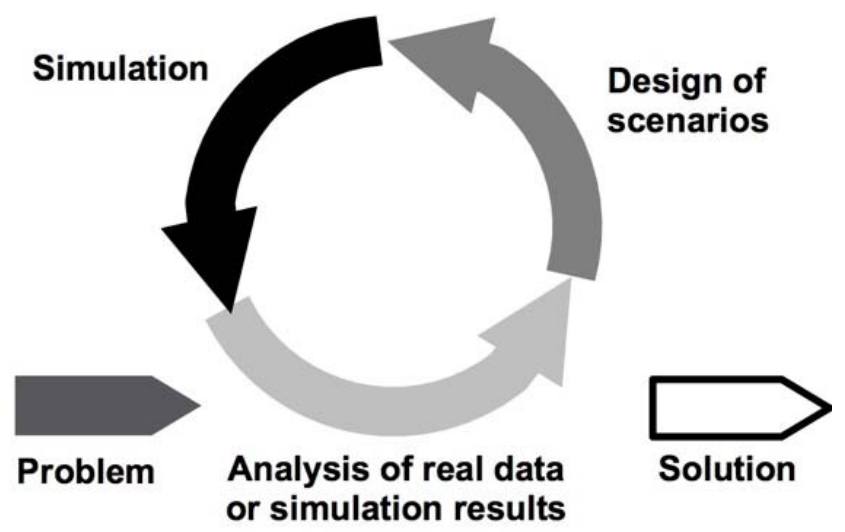

Figure 1: The planning loop.

not anymore sustainable to plan railway services as if they were a deterministic system, since this would cause an over- or underestimation of the effective capacity of the network.

In the planning loop different delay scenarios can be defined, supporting planners in multiple tasks:

- Estimating the reliability that could be obtained with a given timetable;

- Comparing the performances of different timetables;

- Assessing the effects of delay propagation among lines and nodes;

- Assessing the effects of selected delayed services on the other trains;

- Estimating the capacity related to given reliability levels.

1.1 Implementing the planning loop

The planning loop is implemented in TRENO, the planning suite developed by TRENOlab formed by three tools: TRENOAnalysis, TRENOPlus and trenissimo. The characteristics of trenissimo are described in detail in Section 4 of this paper. Fig. 2 shows the planning loop as implemented in the TRENO suite including the key external data sources: the infrastructure model and the train movement data.

TRENOAnalysis is the only tool specifically designed to view and analyse real and simulated traffic data, find critical points and calculate reliability indicators, representing them using a wide range of diagrams and statistics at different aggregation levels. The outcomes of the analysis can be used as input for improving the timetables in TRENOPlus, and as an input for the stochastic simulation of railway operations carried out in trenissimo.

TRENOPlus is a timetable planning tool developed with the aim integrating the conventional planning and the automated generation of timetables [1]. TRENOPlus integrates a macroscopic and the trenissimo microscopic network models to allow estimating running times and therefore planning timetables.

Depending on the available time and data the user can freely decide to use one of the two or even a combination of them. The microscopic model allows not only viewing the usage of lines and stations based on pre-defined headway times, but the blocking time steps are automatically calculated and displayed on the graphic timetable. 


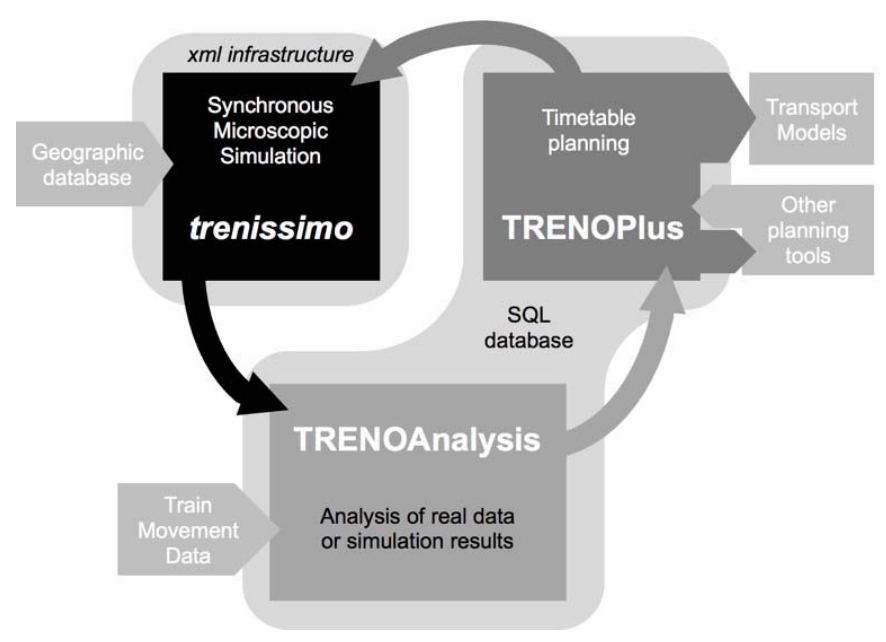

Figure 2: The planning loop as implemented in the TRENO suite.

\section{SIMULATION OF RAILWAY OPERATIONS}

Simulation of railway networks has a long tradition, starting many decades ago in railway laboratories, where models in the scale 1:87 were used to reproduce networks and control them using realistic interlocking systems. The rapid growth in the computational capacity of computers, the creation of graphical user interfaces and the relative simplicity of the basic principles of railway signalling have led to the development of simulation tools towards the end of the 1990s. Simulation tools were first able to simulate relatively small networks considering all trains in a deterministic way. These tools were mainly used to support infrastructure planning and specially to estimate infrastructure capacity. More recently, the further increase in the performance of computers and the possibility of an automatic import of infrastructure layouts and timetables widened the application spectrum of microscopic simulators to large networks and to more detailed stochastic stability evaluations.

Microscopic simulation has gradually become widespread, since it allows considering not only the characteristics of infrastructure, signalling and rolling stock, but also human factors.

\subsection{Types of simulation}

The various types of simulation are presented in detail in [2]. In a microscopic simulation the infrastructure is modelled reproducing the functionality of interlocking, safety and block systems, while in a macroscopic these characteristics are simplified to reduce the computation effort and the required input detail. A simulation is static if it is timeindependent, while a dynamic simulation model shows how a system evolves over time. In continuous simulation models the value of state variables changes continuously in time, therefore it is calculated with analytic continuous resolution of state equations. Different than deterministic model, the stochastic ones contain one or more random components, which are used to reproduce human factors or other variable parameters.

In a standard asynchronous simulation, trains with highest priority are simulated first, and conflicts among them solved with a first-come-first-served strategy; the resulting infrastructure occupations are stored. Then the process is repeated for each priority group, more and more saturating the time-windows which are still unused. Therefore, no conflict 
among trains with different priority is possible: high-priority services are never forced to brake or stop by conflicts with low-priority trains.

On the other hand, in synchronous simulations all trains are calculated together, considering the constraints given by the interlocking and signaling system and the behaviour of drivers. As a result, a synchronous simulation reproduces operations as they are in reality, including the consequences of traffic conflicts: a faster train might be forced to brake behind a lower-priority one, and its driver would follow the braking curve as allowed by the Automatic Train Protection (ATP) system.

Because of these characteristics, the approach that reproduces railway operations in highest detail is dynamic, synchronous, microscopic and stochastic. These models are also the most flexible and widely-used in commercial simulation software as well as in trenissimo.

\subsection{Simulation tools}

A number of synchronous micro-simulation models have been presented in the last years. Since all models use the same approach, solving the motion equation of trains which are moving together on a microscopic network with respect to interlocking and safety system functionality, differences between models are mainly in the accuracy in reproducing the real dispatching and the stochastic components (behaviour of drivers, delays, dwell times, etc), in the flexibility in representing different signaling systems, and in the capability to receive automatic inputs or to generate specific outputs.

The EU funded project Optimal Networks for Train Integration Management across Europe (ON-TIME) recently published an "Assessment of State-of-Art of Train Timetabling" [3], which gives a comprehensive overview on the various commercial simulation software.

The most widely-used commercial software at a global level are OpenTrack [4,5], RailSys [6], RAILSIM X [8] and RTC [9]. Although there is an overlap area in their use, each tool has a different basic philosophy, which makes each of them more suitable for specific applications.

OpenTrack [4] is a simulation tool developed at the Institute for Transport Planning and Systems (IVT) of the ETH Zurich and now supplied and refined by OpenTrack Railway Technology Ltd. [5] is used by railways, the railway supply industry, consultancies and universities in a significant number of Countries. The key strength of OpenTrack compared is the extreme flexibility in defining the infrastructure - simply as nodes and edges using the so-called "double-vertex graph" [9] - and the interlocking and signalling systems. Instead of directly implementing a set of national systems, the tool features a few standardized signals whose characteristics can be customized by the user to model accurately their functions. As a result, basically all signalling systems can be represented, and specific signals can be inserted to influence the behaviour of drivers, making it very realistic. Moreover, at present OpenTrack appears as the tool with the most complete set of stochastic components to represent drivers' behaviour.

RailSys [6] is a comprehensive signal-berth simulation package, which also has modules that facilitate infrastructure management, timetable construction and possession planning. The software database structure allows simple storing of very large models, which is reflected by its use by many infrastructure managers. Refined simulation techniques to prevent deadlocks, a number of input and output capabilities and the presence of variable process and event times made this tool applicable for large-network stochastic simulations.

RAILSIM X [7] developed by SYSTRA, appears as relatively similar to RailSys, but is extremely focused on the US market. It is formed by several modules, which go far beyond 
the pure simulation of operations, or simplify some of its most common uses. These include, among others, the design and analysis of rail and rapid transit signal systems and the analysis of the loads, potentials and regenerative braking receptivity on DC and AC power systems. RAILSIM X is used in particular by passenger rail, light rail and metro operators in the US.

RTC (Rail Traffic Controller) [8] developed by Berkeley Simulation, is the simulation software used by most freight operators in the US to reproduce operations on complex networks, also when formed by long single-track lines that can be used as alternative routes for trains depending on traffic conditions. Instead of using fixed (or multiple, but preselected) itineraries, RTC is able to dispatch trains on the network, finding a deadlock-free routing to their destination. This ability has made RTC a standard trusted by all stakeholders in the US in particular for estimating the capacity of networks considering a set of delay cases as input.

\subsection{Shortcomings of the current simulation tools}

At present, microscopic simulation is the most accurate way to mimic rail operations, since infrastructure, the interlocking and safety systems as well as train movements are represented in considerable detail.

Despite of these strengths, the simulation tools still present a number of weaknesses, which can be grouped in two main categories:

- Limitations caused by the basic principles implemented in a simulation tool;

- General shortcomings of the stochastic simulation.

The four tools listed in 2.2 have a very different origin, which is reflected in the basic principles implemented and thus, as a consequence, in their strengths and weaknesses. Part of these weaknesses are also caused by the limited computational power of computers when the first versions of these tools were released, which for example forced the developers to simplify the models in order to obtain reasonable simulation times.

The details of these weaknesses are not analyzed in detail in this paper; however, we list a few examples. RailSys features an extremely fast simulation algorithm, which allows running several stochastic iterations in a limited time. However, the behavior of drivers is not reproduced correctly, with trains that in most cases follow the timetable with no earlyrunning: instead of driving a train following a certain "driving style", drivers simply follow at the second the planned timetable. At the same time, the dispatcher cannot be configured freely to reproduce the real dispatchers; this weakness is not compensated by an API to allow a real-time communication with external tools. As a result, although it is an extremely powerful and flexible planning suite, RailSys is not able to perform an accurate, state-of-theart synchronous stochastic simulation of rail operations.

At the price of much longer simulation times and a more difficult deployment on very large networks, OpenTrack overcomes these weaknesses by featuring a train-driven simulation process and an API. In the train-driven process trains pre-reserve the block sections in front of them, and the priority is given by default to the first train asking for a certain route (FIFO). Additionally, different rules can be assigned to each signal to prioritize the most delayed train, or the one with the higher-priority category, etc. The API allows controlling nearly all parameters in real time through external tools that can, for example, simulate the corresponding power network or act as a traffic management system. The API makes OpenTrack able to reproduce operations in an extremely high detail; however, this 
detail requires external components such as an external Traffic Management System, which are not easily implemented; moreover, the simulation times increase sharply.

Considering all simulation tools, the key general weaknesses are:

- Stochastic nature of inputs not fully modeled

When the deterministic inputs are set up correctly, the theoretical residual model error is negligible, but the error becomes higher because the stochastic behaviour of some of the inputs is not recognized in the simulation. For example, modelling the dwell times would require a separate consideration of the dwell time due to the passenger exchange and the inaccuracy of the departure process, with the latter being unavoidable also when the train arrives early at a stop. Driving variability is another element that is not fully modelled in the existing commercial simulation tools, in particular concerning the parts of motion in which the behavior of drivers is more variable such as coasting or braking. Some models do not even have the capability to take this into account, while others model them only deterministically.

- Dispatching

Dispatching is strongly connected to the principles considered when implementing a tool. With the exception of the combination of OpenTrack with an external traffic management system (which is not a commercial tool), no other commercial simulation software allows a realistic representation of dispatching. Timetable-driven tools such as RailSys tend to follow the planned timetable quite rigidly, while train-driven tools such as OpenTrack require setting correctly the (often variable) dispatching criteria at a number of signals to prevent the priority to be given to the wrong train. The result is that an on-time stopping train might depart before a late-arriving long-distance train and then delay it significantly on its way, or that an earlyarriving train might enter at a terminal station delaying an on-time departing one.

\section{- $\quad$ Modelling major disruptions}

The existing simulation tools - with the exception of OpenTrack with an external Traffic Management System - do not cancel services automatically, often leading to unrealistically high output delays when simulating significant initial delays or disruptions. A further issue with simulating major disruptions is that rolling stock and/or train crew reallocation become critical parts of the problem. No microscopic simulations currently deal with these and ignoring them can lead to simulators significantly under- or overestimating the impact on performance of particular scenarios. As major disruptions represent a significant proportion of total train lateness, this seriously limits the usefulness of microscopic simulation to estimate delays ex-ante. Thus, the possibility to control simulation using external tools or the integration of realistic dispatching algorithms are a key area for development of microscopic simulation packages in order to meet more fully the needs of railway operators and planners.

\section{TRENISSIMO}

Trenissimo is based on the experience gained in several years of activity with the stochastic simulation both at an academic and professional level [2], and was developed with the specific aim of overcoming the weaknesses listed at the previous section, obtaining a tool that: 
- Is perfectly integrated in a suite to cover all stages of planning, with the advantages of using a set of dedicated - and thus optimized for their use - tools but without the disadvantage of moving files among tools.

- Can be used across on computers with all operating systems.

- Combines the highest accuracy with reasonable computation times, using all resources available on multi-core computers.

- Can represent correctly any interlocking, signaling and ATP/ATC system, including all signal aspects and the corresponding behavior of drivers.

- Allows a realistic dispatching of trains without the need to use external tools.

- Represents the human factors and other stochastic components with the highest accuracy in particular concerning the dwell and running times.

- Is suitable to perform stochastic sensitivity analysis considering the combined impact of multiple parameters, such as delays, margins, headway times, etc.

- Is compatible with the standard RailML infrastructure format.

- Can consider correctly the relationship between number of passengers and dwell time, in particular in high-frequency services.

trenissimo is a Java application natively compatible with all operating systems. It was developed using the Netbeans Platform, a framework designed to create a very flexible and user-friendly environment: it permits to work with graphical elements and dispose them in different monitors, grouping them together into tabs, etc. Netbeans Platform is used in a number of complex professional applications developed and/or used among others at Oracle, Northrop Grumman, Boeing, NASA, NATO and, in the field of simulation transport system, AnyLogic.

\subsection{Input data}

The input data are separated into 6 groups:

- Rules: the general rules/settings of a country/network: speedtypes, signal aspects /ATP /ATC, power supply.

- Macroscopic infrastructure: each OCP is represented by a point, and the lines are formed by simple edges connecting them. This macroscopic network creates the perfect compatibility with the TRENOPlus planning tool, and can be imported from it.

- Microscopic infrastructure: the microscopic network, including the alignment, the complete layout of stations, speed limits, signalling system, etc.

- Simulation data: the simulation area (in case it is a portion of the network model) and its corridors.

- Rolling Stock: the technical characteristics of trainsets.

- Timetable: in a very simple format produced by TRENOPlus that requires no import to be used.

The combination of these inputs forms a project: by changing any of them within the tool a new project is generated, and only the modified data are duplicated, while all the others are maintained. The navigation among all projects (scenarios) is extremely intuitive and simple, allowing the users to easily create and store several variants. 


\subsection{Graphical editor}

The graphic editor for the infrastructure uses the well-known double vertex graph to represent the network [10]. This characteristic allows a very intuitive editing of the infrastructure and simplifies the creation of train routes. Moreover, it gives the highest flexibility in representing the specific characteristics of each station and line, without forcing the use of pre-defined blocks. This allows, for example, the presence of different OCPs on the two tracks of a double track line.

It is possible to import the infrastructure from RailML 2.3 files. The infrastructure can be edited graphically as drag\&drop of elements or imported as csv table. All characteristics of all elements are shown in tabs. The tool allows finding any element by entering part of its characteristic(s) (such as the ID for a station), as well as highlights elements with missing relevant characteristics (such as the speed). A Navigator allows easily navigating in large files, while a Macroscopic Network view can be used to quickly open the microscopic model of any station or part of the network.

\subsection{Simulation and dispatching}

The tool reproduces railway operations in a mixed continuous-discrete approach: it calculates the solution well-known motion equation [11] of trains in a continuous way, considering the discrete processes of signal states. At present, trenissimo features the Italian, French, British and Norwegian signaling systems, as well as the ETCS Level 1 and 2; other systems including the CBTC and ETCS Level 3 - will be implemented in the coming months.

One of key strengths of trenissimo is that also the dispatcher is simulated: as in the real world, while automatic block signals are automatically set to green, a dispatcher oversees the operation, opening the home and exit signals based on the planned and actual positions of trains. As a result, and similar to real operations, the dispatcher always controls operations: he can take decisions based on simple rules, or more complex algorithms. This principle allows implementing robust deadlock-prevention algorithms, as well as testing the effective impact of different dispatching strategies. Based on a set of rules, the dispatcher is also able to cancel train services, or short-turning them the reduce the propagation of delays.

\subsection{Stochastic parameters}

Following the principles explained in [2], the key stochastic inputs for an accurate simulation are the initial delays, the dwell times and the variability of running times. trenissimo fully implements the combination of stochastic dwell time and departure inaccuracy proposed in [12] to accurately represent the dwell times of the early- and late-arriving trains.

Concerning the variability of running times, based on the results of an analysis of train tracking data [13], in trenissimo the driving style of drivers is represented by a set of six parameters, one for each phase of motion: acceleration, cruising, coasting, braking at stops, signals and speed restrictions.

\subsection{Dwell times and passengers}

In high-frequency services in urban areas such as on metro lines passengers do not reach the stop based on the timetable: they simply take the first train that reaches their destination. As a result, the number of passengers that are waiting for a train depends on the actual headway from the previous train and is an independent stochastic variable. 
Given an O/D matrix and a relationship between number of passengers boarding/alighting and on board and dwell time trenissimo simulates the increasing number of passengers waiting for a train on all platforms and estimates the corresponding dwell time. This appears extremely important to reproduce the longer dwell time of metro and suburban trains arriving late and thus further increasing their delay.

\subsection{Outputs}

Besides a recorded animation of the simulation that shows the trains moving on the network during the simulation including the exact signal aspects, trenissimo produces all classic graphic timetables, statistics and outputs. The graphic timetables can be visualized using the so-called stochastic blocking times [14], in which the blocking times of several iterations of a Monte Carlo simulation are represented on the same diagram using transparencies: the darker a set of blocking times is, the lower the variability of the corresponding service. Also the occupation areas of different courses can overlap: the darker overlapping area, the higher is the probability of conflict. Fig. 3 shows an example of the stochastic blocking times.

Besides its integrated viewer, the results of stochastic simulation can be imported and analysed with the wider and attractive set of diagrams and statistics produced by TRENOAnalysis which, additionally, allows directly comparing real and simulated data as well as the result of multiple scenarios.

\subsection{Other technical characteristics}

In order to reduce the usage of RAM memory and the computation times, command line execution of simulations in trenissimo is also available. The user may use this execution mode to execute multiple simulations simultaneously. This allows to take advantage of modern multi-core CPUs, optimising their usage and automating operations such as multivariate analysis (e.g., scenarios with increasing initial delays). Additionally, when running Monte Carlo simulations, the workload will be divided into multiple tasks, each assignable to a different workstation of a network.

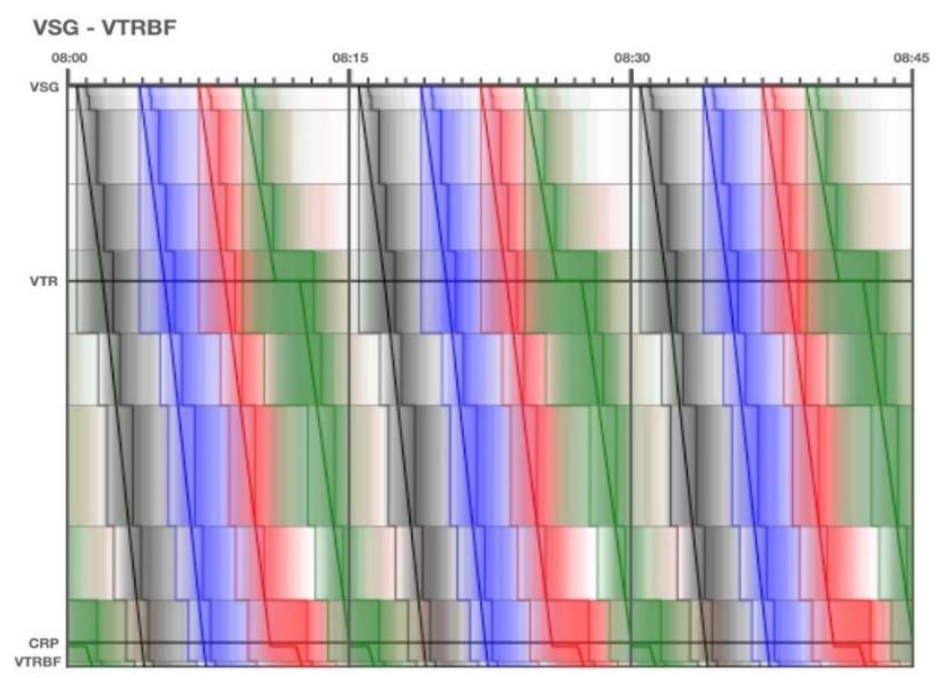

Figure 3: Stochastic blocking times, as visualized in trenissimo. 
In a later release, trenissimo will also support the versioning of infrastructure files, greatly simplifying the management of incremental improvement plan and other studies that require developing and testing several infrastructure configurations.

\section{APPLICATION FIELDS OF TRENISSIMO}

The aim of trenissimo was to focus on some applications suitable for the academia and railway undertakings.

Considering its key characteristics, trenissimo appears most suitable when the highest detail in the stochastic simulations is needed, when a realistic dispatching plays an important role, or when a large number of scenarios has to be developed and tested.

\subsection{Stochastic simulation of metro networks}

The explicit consideration of the actual number of passengers in the estimation of dwell times, combined with the detailed modelling of signaling systems, allow simulating metro operations with a higher accuracy compared to the other commercial tools.

\subsection{Combined analysis - planning - simulation studies}

When the loop formed by the analysis of operational data, timetable planning and simulation has to be repeated multiple times, the combination of trenissimo, TRENOPlus and TRENOAnalysis appears as a unique combination that guarantees the maximum efficiency. No file has to be imported and exported between the tools, and the definition of alternative scenarios is made interactively: as a result, the time spent is remarkably reduced, together with the probability of errors.

\subsection{Ex-ante estimation of delays/performance modelling}

The ex-ante estimation of delays is one of the most demanding tasks for a microscopic simulation tool, since it must reproduce with the highest possible accuracy all phenomena involved in railway operations. At the same time, it appears as one of the most interesting estimations for railway undertakings, since it allows estimating the performances of a timetable not only qualitatively or as relative comparison, but obtaining the same key indicators used to measure real operations.

\subsection{Sensitivity analysis}

In particular in high-level or academic studies it is required to test the impact of the combined variation of a number of input parameters. This can be, for example, the trade-off between capacity usage and reliability of services, also when combined with additional parameters such as margins or input delays. This analysis appears to be very important in particular to support the design of infrastructures, or the activation of major investments: allowing a precise estimation of the robustness of operations at increasing traffic, delays, number of passengers or other factors is key to dimension the investments at best, avoiding unnecessary expenses.

trenissimo is ideal to use the so-called "stochastic UIC406" [14] method, which overcomes the limitations of the well-known UIC406 method [15] for estimating the capacity of railway networks. Similar to the UIC406, the method requires a "compressed" timetable as an input: margins, delays and supplements are then inserted incrementally and the stochastic simulation of each combination of them performed. The resulting performance 
indicators at increasing margins, supplements and delays allow graphically representing the trade-off between capacity and performances on a line and thus identifying not the capacity as function of the performance targets.

\section{FIRST TESTS}

The first comparison tests of the new tool have been carried out on two different lines that were previously modelled by the Authors: the Lyon-Grenoble in France and the LondonShenfield in the UK.

\subsection{Lyon-Grenoble}

The Lyon-Grenoble is a mixed-traffic double-track line on the French national. With over than $70 \mathrm{TGV}, 130 \mathrm{IC}$ and regional trains and a dozen of freight trains it is the most important in the Region with approximately 22,000 passengers per day. The operations are complex due to the junctions of Moirans (line to Valence), St-André-le-Gaz (single-track line to Chambery) and Grenay (connection to the Paris-Marseille HSL).

On this line the tool was used to perform the stochastic simulation of the current operations of a working day, using as input the real initial delay, dwell times and train performance distributions. The test was performed using trenissimo with its innovative dispatcher functionalities (SIM OP), then with a train-driven approach (SIM TR), and a timetable-driven approach (SIM TT).

The differences between the three simulations appear in diagram in Fig. 4, which shows the comparison of real (plain line) and simulations of the Regional trains of the morning peak hour between Grenoble and Lyon. Due to the numerous dispatching decisions and strong augmentation of the delays on the line, the new operation approach of trenissimo (SIM OP) leads to a more accurate reproduction of real delays. In the train-driven approach (SIM TR), which includes a significant important pre-reservation of the blocks head, tends to improve

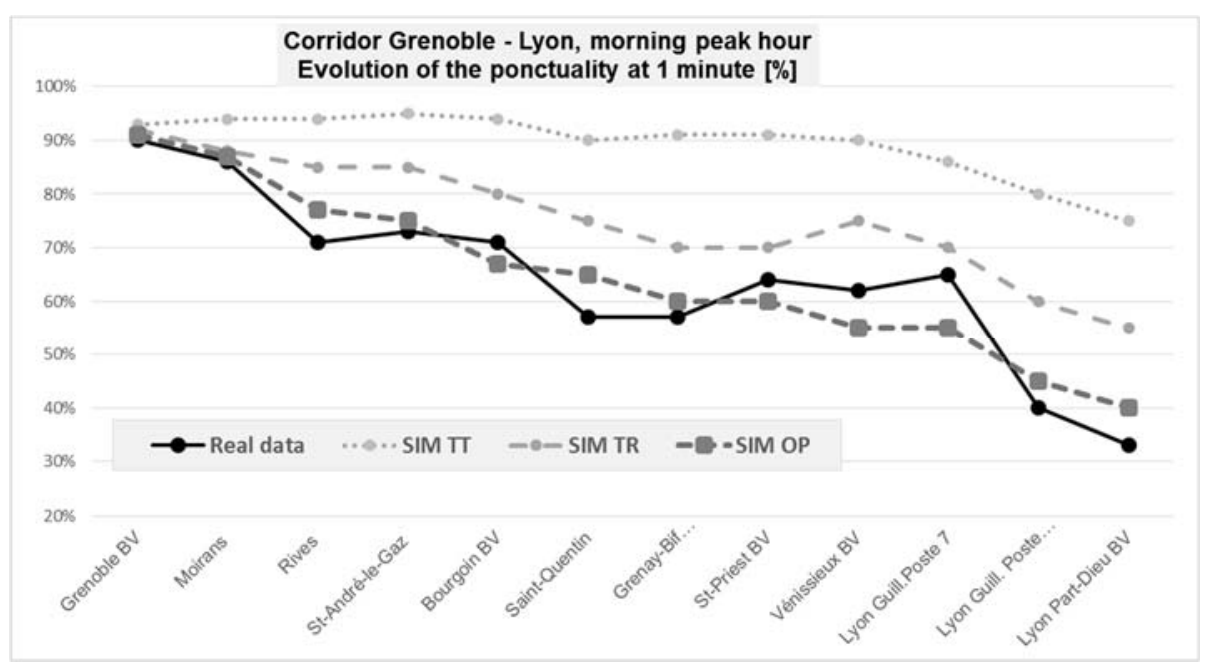

Figure 4: Comparison of real data and simulation approaches on the Lyon-Grenoble corridor (France). 
the advancement of trains compared to reality. In the timetable-driven approach (SIM TT), the tool tends to maintain the planned slots for all trains with highest accuracy leads to a remarkable error, underestimating the delays for this category of trains.

\subsection{London-Shenfield}

The London-Shenfield line is a double-track commuter line that connects the major Liverpool Street terminal with Shenfield, located around $30 \mathrm{~km}$ east of London. The line is mostly used by commuters, and thus is characterized by an even service -a train every 10 minutes- for most of the day, and a sharp increase in the peaks, with headways of just 2 ' in some cases. In this case, the dwell times are extremely important to reproduce correctly the operations situation and obtain a realistic estimation of any timetable or operational change.

In the London-Shenfield, the simulation of the line with trenissimo was performed to evaluate the impact of a new timetable, as well as to tests the effects of an improved representation of dwell times. They are estimated as function of the actual number of passengers waiting for the train on the platform and the passenger loadings in the train, and thus allows a more realistic reproduction of the impact of the variable headway times on the dwell times, and thus on delays. The key input for this function, the number of passengers/minute accessing each station during the day, was derived from passengers counts performed for all trains and at all stations.

Fig. 5 shows the punctuality at 3' at arrival at Liverpool Street station for the sequence of trains during the morning peak. Compared to the real data of April 2016, the simulation with standard dwell times function (no PAX) shows a small punctuality increase, which would not clearly justify a timetable change.

But the simulation with passenger function (with PAX), taking correctly in account the new dwell-time, shows a significant better result. Based on this estimation, the timetable change was introduced in May 2016: the real data of the first month of operation of the new timetable show not only a remarkable improvement compared to the previous month, but also show a trend similar to the PAX simulation.

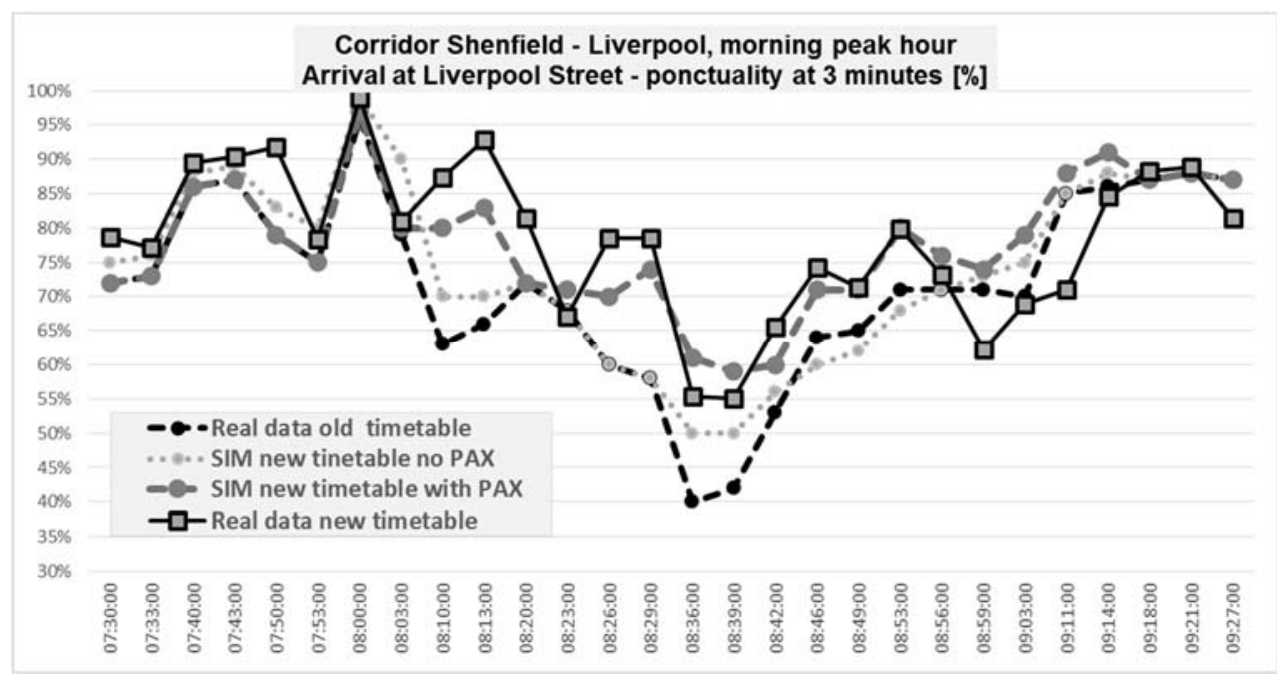

Figure 5: Trenissimo results on the Shenfield-London line (UK). 


\section{CONCLUSIONS}

Based on years of experience in the simulation of railway operations, trenissimo was developed to make the simulation more accurate and easier-to-use at the same time.

A special focus was given to two major weaknesses of the most widely-used commercial tools: the dispatching of trains and the representation of the stochastic parameters. Additionally, trenissimo takes full advantage of a state-of-the art development framework and of technologies not used in this field yet.

The first tests, performed on two remarkably different lines show encouraging results: the simulation method of trenissimo shows a better reproduction of real operations, demonstrated by the lower difference between real data and output delays.

\section{REFERENCES}

[1] de Fabris, S., Longo, G., Medeossi, G. \& Pesenti, R., Automatic generation of railway timetables based on a mesoscopic infrastructure model. Journal of Rail Transport Planning \& Management, 4, pp 2-13, 2014.

[2] Medeossi, G. \& de Fabris, S., Simulation of rail operations. Handbook of Optimization in the Railway Industry, Springer, pp. 1-24, 2018.

[3] ON-TIME Project Task 3.1 - Assessment of state-of-art of train timetabling, Online. ontime-project.eu. Accessed on: 19 May 2018.

[4] Nash, A. \& Huerlimann, D., Railroad simulation using OpenTrack. Computers in Railways $I X$, eds. J. Allan, R.J. Hill, C.A. Brebbia, G. Sciutto \& S. Sone, WIT Press, pp. 775-784, 2004.

[5] OpenTrack, OpenTrack Railway Technology, Online. opentrack.ch. Accessed on: 19 May 2018.

[6] RailSys - RMCon, Online. rmcon.de. Accessed on: 19 May 2018.

[7] Rail Traffic Controller - Berkeley Simulation Software, Online. berkeleysimulation.com. Accessed on: 19 May 2018.

[8] Montigel, M., Modellierung und Gewährleistung von Abhängigkeiten in Eisenbahnsicherungsanlagen. $\mathrm{PhD}$ thesis, ETH Zürich, 1994.

[9] Wende, D., Fahrdynamik, Teubner, Stuttgart, 2003.

[10] Longo, G. \& Medeossi, G., Enhancing timetable planning with stochastic dwell time modelling. Computers in Railways XIII, WIT Press: Southampton, pp. 461-471, 2012.

[11] de Fabris, S., Longo, G. \& Medeossi, G., Automated analysis of train event recorder data to improve micro-simulation models. Computers in Railways XI, J. Allan, E. Arias, C.A. Brebbia et al., WIT Press: Southampton, pp. 575-585, 2008.

[12] Medeossi, G., de Fabris, S. \& Longo, G., A method for using stochastic blocking times to improve timetable planning. Journal of Rail Transport Planning \& Management, 1, pp 1-13, 2011.

[13] Medeossi, G., Capacity and Reliability on Railway Networks, A Simulative Approach. $\mathrm{PhD}$ thesis, University of Trieste, 2010

[14] UIC, Leaflet 406 - Capacity, 3rd ed., International Union of Railways (UIC), 2012. 